\title{
DECOMPOSITION NUMBERS FOR SPIN CHARACTERS OF EXCEPTIONAL WEYL GROUPS OF TYPE $E_{n}$
}

\author{
By Muhammad SALEem
}

\section{Introduction}

It is well known that the groups, $W\left(E_{6}\right) \cong S_{4}(3) .2 \cong U_{4}(2) .2 ; W\left(E_{7}\right) \cong C_{2} \times$ $B_{3}(2) \cong C_{2} \times D_{7}(2)$, where $C_{2}$ is the cyclic group of order 2 and $W\left(E_{8}\right) \cong 2 . O_{8}^{+}(2)$. $2 \cong 2 . D_{4}(2) .2$ (see, $[\mathrm{CCN}]$ ),

In this paper, which is a continuation of $[\mathrm{Sa}]$, the decomposition matrices for the spin characters of the exceptional Weyl groups of type $E_{n}(n=6,7,8)$, for the prime numbers $p=5,7$ are determined. In all cases except $W\left(E_{8}\right)$ and $p=5$, the relevent prime number divides, the order of the group to the first power only.

First we use the central characters to split the ordinary characters into $p$ blocks (For general information about $p$-blocks, see $[\mathrm{CR}]$ ). Let $B$ be a $p$-block of a group $G$ and let $\Psi_{1}, \cdots, \Psi_{s}$ and $\phi_{1}, \cdots, \phi_{r}$, respectively, denote the ordinary and $p$-modular irreducible characters of $G$ in $B$. The restriction of each $\Psi_{\imath}$ to $p$-regular classes of $G$, denoted by $\bar{\Psi}_{2}$ is a $p$-modular character of $G$ and $\bar{\Psi}_{\imath}=\sum_{j=1}^{r} d_{\imath j} \phi_{j}(1 \leqq i \leqq s)$ where $d_{\imath \jmath}$ 's are non-negative ingers, called the decomposition numbers of $B$ for the prime $p$. The $(s \times r)$ matrix $D_{P}^{B}(G)=\left(d_{\imath j}\right)$ is called the decomposition matrix of $B$ for the prime $p$. Furthermore, a principal character $\sum_{\imath=1}^{s} c_{i} \Psi_{\imath}$ will be identified with the column of integers $c=\left(c_{\imath}\right)$ and it may be indecomposable or sum of principal indecomposable characters in $B$.

One way to constructs $D_{P}^{B}(G)$ is to find the principal indecomposable characters in $B$. This method is described by James and Kerber [JK] and is as follows; suppose that the matrix

$$
R_{P}^{B}(G)=\left(c^{(0)}, c^{(1)}, \cdots, c^{\left(n_{1}\right)}, \cdots, c_{r-1}^{(0)}, c_{r-1}^{(1)}, \cdots, c_{r-1}^{\left(n_{r-1}-1\right)}, c_{r}\right)
$$

of principal characters has been found such that, with a suitable arrangement of the ordinary irreducible characters, for $0 \leqq k_{\imath} \leqq n_{\imath}$ and $i=1, \cdots,(r-1)$, each of the matrices

$$
R_{P}^{B}(G)=\left(c_{1}^{\left(k_{1}\right)}, \cdots, c_{r-1}^{(k r-1)}, c_{r}\right)
$$

has the triangle of zeros above the main diagonal and 1's on the main diagonal.

Received October 30, 1992; revised August 23, 1993. 
Then $D_{P}^{B}(G)$ has the same shape as $R_{P}^{B}(G)$; the entries in $R_{P}^{B}(G)$ are greater than or equal to the corresponding entries in $D_{P}^{B}(G) ; d_{r}=c_{r}$ and for any $\imath=1$, $\cdots, r-1 ; k_{\imath}=0,1, \cdots, n_{\imath}$

$$
d_{\imath}=c_{j}^{\left(k_{\imath}\right)} \longrightarrow \sum_{j=1+\imath}^{r}\left(a_{j}^{\left(k_{i}\right)}\right) d_{\jmath}
$$

where $a_{\jmath}^{\left(k_{i}\right)}$ are non-negative integers and $d_{\imath}(\imath=1, \cdots, r)$ are the columns of $D_{P}^{B}(G)$.

If $G$ is a group, $p$ a prime then the symbols $\operatorname{irr}(G), \operatorname{irm}_{p}(G), \operatorname{indec}_{p}(G)$ denote the full set of ordinary irreducible, $p$-modular irreducible and principal $p$-indecomposable characters of $G$, respectively. Similarly, the full set of irreducible spin, $p$-modular spin and principal $p$-indecomposable spin characters of $G$ are denoted respectively by $\operatorname{sirr}(G), \operatorname{sirm}_{p}(G)$ and $\operatorname{sindec}_{p}(G)$. If there is no confusion about $p$, then we will omit it. Furthermore $\uparrow G(\downarrow G)$ denote the induction from (restriction to) a subgroup of $G$ and the symbol $\phi$ (least degree character of corresponding column) denote the $p$-modular irreducible character corresponding to that column in the matrix.

\section{1. $\boldsymbol{W}\left(\boldsymbol{E}_{6}\right), p=5$}

There are 11 elements in $\operatorname{sirm}\left(E_{6}\right)$, which are distributed over ten 5-blocks. The elements $40_{s}, 40_{s s}, 120_{s}, 60_{s}, 60_{s s}, 80_{s}, 80_{s s}, 20_{s}$ and $20_{s s}$ of $\operatorname{sirr}\left(E_{6}\right)$, each form a 5-block on its own. There is also 5-block of full deffect containing $8_{s}$, $72_{s}, 64_{s}, 64_{s s} \in \operatorname{sirr}\left(E_{6}\right)$, the later two being exceptional for $p=5$, (see, [Mo, p. 578]) and hence the Brauer tree is as follows, (see, [Fe; Theorem 9.2; p. 307]),

$$
8 s-72_{s}-64_{s}=64_{s s} \text {. }
$$

\section{2. $\boldsymbol{W}\left(\boldsymbol{E}_{7}\right), \boldsymbol{p}=\mathbf{5}$}

In this case, the elements of $\operatorname{sirr}\left(E_{7}\right)$ are distributed into ten 5-blocks. The following $280_{s}, 720_{s}, 560_{s}, 120_{s}, 280_{r}, 720_{r}, 560_{r}$ and $120_{r}$ are those elements of $\operatorname{sirr}\left(E_{7}\right)$, whose degrees are divisible by 5 , each form its own block are in $\operatorname{sindec}\left(E_{7}\right)$ and are also belonging to $\operatorname{sirm}\left(E_{7}\right)$.

There remain four blocks (with full defect) the first two blocks each contains four elements of $\operatorname{sirr}\left(E_{7}\right)$, whereas the third and fourth blocks each contains five elements of $\operatorname{sirr}\left(E_{7}\right)$ namely:

$$
\begin{aligned}
& B_{1}: 48_{s}, 112_{s}, 64_{s} \text { and } 64_{s s} \\
& B_{2}: 48_{r}, 112_{r}, 64_{r} \text { and } 64_{r r} \\
& B_{3}: 8_{s}, 168_{s}, 112_{s s}, 512_{s} \text { and } 448_{s} \\
& B_{4}: 8_{r}, 168_{r}, 112_{r r}, 512_{r} \text { and } 448_{r} .
\end{aligned}
$$


On consideration of degrees, the Brauer trees of the block $B_{1}$ and $B_{2}$ are respectively, therefore as follows;

$$
\begin{aligned}
& 48_{s}-112_{s}-64_{s}=64_{s s} \\
& 48_{r}-112_{r}-64_{r}=64_{r r} .
\end{aligned}
$$

We now determine the decomposition matrices corresponding to the block $B_{3}$ and $B_{4}$.

Inducing the elements $40_{s s}, 120_{s}, 40_{s}$ of $\operatorname{sindec}\left(E_{6}\right)$ to $W\left(E_{7}\right)$, we get the following decompositions.

and

$$
\begin{aligned}
40_{s s} \uparrow E_{7}= & 112_{s s}+560_{s}+448_{s}+112_{r r}+560_{r}+448_{r} . \\
120_{s} \uparrow E_{7}= & 280_{s}+512_{s}+2.720_{s}+560_{s}+448_{s}+120_{s} \\
& +280_{r}+512_{r}+2.720_{r}+560_{r}+448_{r}+120_{r} .
\end{aligned}
$$

$$
\begin{aligned}
40_{s} \uparrow E_{7}= & 48_{s}+168_{s}+280_{s}+112_{s}+512_{s} \\
& +48_{r}+168_{r}+280_{r}+112_{r}+512_{r} .
\end{aligned}
$$

Now considering the block decomposition of characters, the direct summand of elements of $\operatorname{sindec}\left(E_{7}\right)$ will imply the connection of some pairs of characters in the Brauer trees corresponding to the block $B_{3}$ and $B_{4}$ which together with the fact that $8_{s}\left(8_{r}\right)$ and $168_{s}\left(168_{r}\right)$ are both congruent to -2 (modulo 5 ) is enough to uniquely determine the Brauer trees for $B_{3}$ and $B_{4}$ as given by:

$$
8_{s}-112_{s s}-448_{s}-512_{s}-168_{s}
$$

and

$$
8_{r}-112_{r r}-448_{r}-512_{r}-168_{r} .
$$

\section{3. $\boldsymbol{W}\left(\boldsymbol{E}_{8}\right), \boldsymbol{p}=\mathbf{5}$}

Here $\left|\operatorname{sirm}\left(E_{8}\right)\right|=23$, and they are distributed over 115 -blocks. Each of the elements $5600_{s}, 4800_{s}, 5600_{s s}, 800_{s}, 2800_{s s}, 2800_{s}, 5600_{s s s}, 11200_{s}$ and $8400_{s}$ of $\operatorname{sirr}\left(E_{8}\right)$ forms a block on its own and the elements $320_{s}, 1120_{s}, 6720_{s}, 6480_{s}$, $1680_{s} \in \operatorname{sirr}\left(E_{8}\right)$ lies in a block of defect 1. Furthermore, the Brauer tree of this block is:

$$
320_{s}-1680_{s}-6720_{s}-6480_{s}-1120_{s} .
$$

Since the spin principal characters $\left(112_{s}+64_{s}+64_{s s}\right) \uparrow E_{8}, 560_{s} \uparrow E_{8}$, and $\left(112_{s s}+\right.$ $\left.448_{s}\right) \uparrow E_{8}$, induced from $W\left(E_{7}\right)$ gives the connection of $320_{s}$ with $1680_{s} ; 6720_{s}$ with $6480_{s}$ and $6480_{s}$ with $1120_{s}$ and this can be seen from their respective decompositions given below:

$$
320_{s}+448_{s}+1680_{s}+2592_{s}+5600_{s s}+9072_{s}+7168_{s}+2.8192_{s}+2.7168_{s s} .
$$




$$
\begin{aligned}
& 2592_{s}+2.5600_{s}+2.4800_{s}+2.9072_{s}+2800_{s}+7168_{s}+1120_{s}+2.8400_{s} \\
& +2.11200_{s}+6480_{s}+2.8192_{s}+2016_{s s}+2016_{s s s}+2.7168_{s s}+1344_{s} .
\end{aligned}
$$

And

$$
\begin{aligned}
& 448_{s}+224_{s}+2592_{s}+1344_{s}+5600_{s}+2.4800_{s}+2.9072_{s}+3.8400_{s}+2.11200_{s} \\
& +6720_{s}+6480_{s}+2.8192_{s}+2016_{s s}+2016_{s s}+2.7168_{s}+896_{s} .
\end{aligned}
$$

The remaining elements of $\operatorname{sirr}\left(E_{8}\right)$ belong to a principal block. Since 13 elements of $\operatorname{sirm}\left(E_{8}\right)$ has been determined therefore the principal block contains 10 elements which we have to determined yet.

Let $\operatorname{sindec}\left(E_{7}\right)=\left\{\phi_{1}, \cdots, \phi_{20}\right\}$ and $\operatorname{sindec}\left(S_{9}\right)=\left\{\S_{1}, \cdots, \S_{6}\right\}$ (see [Ya] for $\left.D_{\langle 9,5\rangle}\right)$, as given in the Appendix. In $[\mathrm{KM}]$, it has been shown that $56_{P}, 28_{P}$, $8_{P} \in \operatorname{irm}\left(E_{8}\right)$.

Now consider the following principal spin characters of $W\left(E_{8}\right)$ which are formed by inducing from $W\left(E_{7}\right), S_{9}$ and taking the products of characters as appropriate and then restricting them to the principal block. We obtain the matrix $R_{5}^{B_{1}}\left(E_{8}\right)$; where the columns correspond to $c_{1}=\phi_{1} \uparrow E_{8}, c_{1}^{\prime}=\S_{1} \uparrow E_{8}, c_{2}=$ $\left.\phi_{5} \uparrow E_{8}, \quad c_{3}=\phi_{6} \uparrow E_{8}, \quad c_{4}=\left(\S_{2} \uparrow E_{8}\right) / 2, \quad c_{5}=8_{P} \otimes\left[1680_{s}+6720_{s}\right], \quad c_{6}=\S_{6} \uparrow E_{8}\right) / 2, \quad c_{6}^{\prime}=$

\begin{tabular}{|c|c|c|c|c|c|c|c|c|c|c|c|c|}
\hline $16_{s}$ & 1 & 1 & & & & & & & & & & \\
\hline $112_{s}$ & 1 & & 1 & & & & & & & & & \\
\hline $448_{s}$ & 1 & & 1 & 1 & & & & & & & & \\
\hline $224_{s}$ & 1 & 2 & & & 1 & & & & & & & \\
\hline $448_{s s}$ & 1 & 1 & 1 & & & 1 & & & & & & \\
\hline $896_{s}$ & & 2 & & & 2 & & 1 & 1 & & & & \\
\hline $1344_{s}$ & 1 & 2 & & & 1 & & & & 1 & & & \\
\hline $1344_{s s}$ & 1 & 2 & 1 & & & & & & & 1 & & \\
\hline $2016_{s s}$ & & 2 & & & 2 & & 1 & & & & 1 & \\
\hline $9072_{s}$ & 1 & 6 & 1 & 1 & 4 & & 3 & 4 & 1 & 1 & & 1 \\
\hline $2592_{s}$ & 1 & 2 & 1 & 1 & 1 & 1 & 1 & & & & 1 & \\
\hline $2016_{s}$ & & 2 & 1 & & 1 & & 1 & 1 & & 1 & & \\
\hline $7168_{s}$ & & 3 & 1 & 1 & 1 & 1 & 2 & 3 & & 1 & & 1 \\
\hline $8192_{s}$ & & 4 & & 2 & 3 & 1 & 3 & 3 & & & 1 & 1 \\
\hline $2016_{s s s}$ & & 2 & & & 2 & & 1 & 1 & 1 & & & \\
\hline $7168_{s s}$ & & 2 & & 2 & 2 & & 2 & 3 & 1 & & & 1 \\
\hline & $c_{1}$ & $c_{1}^{\prime}$ & $c_{2}$ & $c_{3}$ & $c_{4}$ & $c_{5}$ & $c_{6}$ & $c_{6}^{\prime}$ & $c_{7}$ & $c_{8}$ & $c_{9}$ & $c_{10}$ \\
\hline
\end{tabular}
$28_{P} \otimes 8400_{s}, \quad c_{7}=\phi_{9} \uparrow E_{8}, \quad c_{8}=8_{P} \otimes 4800_{s}, \quad c_{9}=28_{P} \otimes 2800_{s}$ and $c_{10}=\left(\phi_{8} \uparrow E_{8}\right) / 2$ respectively, given below :

$$
R_{5}^{B_{1}}\left(E_{8}\right)
$$


The final colum $c_{10} \in \operatorname{sindec}\left(E_{8}\right)$, since no subsum of $9072_{s}+7168_{s}+8192_{s}+$ $7168_{s s}$, is congruent to zero $(\bmod 25)$ and we denote it by $d_{10}$. Also by the same argument, $c_{9}, c_{8}, c_{7}, c_{5} \in \operatorname{sindec}\left(E_{8}\right)$ and therefore $d_{\imath}=c_{\imath}$, for $i=5,7,8,9$.

Then the possibilities for $c_{6}$ are $d_{6}=c_{6}-\alpha \cdot d_{9}-\beta \cdot d_{10}$, where $\alpha=0$ or 1 and $\beta \in[0,1,2]$. Now, since $c_{11,6}=1$ and $c_{11,6}^{\prime}=0$, where $c_{\imath, 3}$ has the usual meaning of matrix entries and therefore $c_{b}^{\prime \prime}:=c_{6}-d_{9}$ is a principal spin character. We consider later whether any copies of $d_{10}$ can be subtracted from $c_{6}^{\prime \prime}$.

The columns $c_{6}^{\prime \prime}, d_{7}, d_{9}$ and $d_{10}$ may be subtracted from $c_{4}$ once each. Whatever is the $d_{4}$, the possibilities for $c_{3}$ are $d_{3}=c_{3}$ or $c_{3}-d_{10}$. It can be verified that

$$
\begin{aligned}
28_{P} \otimes 5600_{s}= & 448_{s}+1680_{s}+2592_{s}+2.1344_{s}+5.5600_{s}+8400_{s} \\
& +6480_{s}+4800_{s}+2.5600_{s s}+2.11200_{s}+2.6720_{s} \\
& +2.9072_{s}+8192_{s}+2.2016_{s s s}+3.7168_{s s}+2800_{s},
\end{aligned}
$$

and hence

$$
P_{1}:=448_{s}+2592_{s}+2.1344_{s}+2.9072_{s}+8192_{s}+2.2016_{s s s}+3.7168_{s s}
$$

as a sum of elements of $\operatorname{sindec}\left(E_{8}\right)$ in the principal block of $W\left(E_{8}\right)$. However, $P_{1}=c_{3}+2 \cdot d_{7}-d_{10}$ and this implies that $d_{10}$ is a component of the column $c_{3}$. Thus we put $d_{3}=c_{3}-d_{10}$. Clearly $d_{3} \in \operatorname{sindec}\left(E_{8}\right)$, by the usual subsum argument. Now the possibilities $d_{2}=c_{2}$ or $c_{2}-d_{8}$. It can be checked that

$$
\begin{aligned}
56_{P} \otimes\left[320_{s}+1680_{s}\right]= & 16_{s}+3.112_{s}+4.320_{s}+224_{s}+3.448_{s s}+5.1680_{s} \\
& +4.2592_{s}+2016_{s s}+1344_{s}+2.5600_{s}+4800_{s} \\
& +2.5600_{s s}+9072_{s}+1120_{s}+3.2800_{s}+11200_{s} \\
& +6720_{s}+1344_{s s}+6480_{s}+8192_{s}+5600_{s s}
\end{aligned}
$$

and hence

$$
\begin{aligned}
P_{2}:= & 16_{s}+3.112_{s}+3.448_{s}+224_{s}+3.448_{s s}+2016_{s s}+1344_{s} \\
& +9072_{s}+8192_{s}+1344_{s s}+4.2592_{s}
\end{aligned}
$$

as a sum of elements in $\operatorname{sindec}\left(E_{8}\right)$ in the principal block of $W\left(E_{8}\right)$. But $P_{2}=$ $c_{1}+2 \cdot c_{2}-2 \cdot d_{8}+d_{9}$ implies that $c_{2}-d_{8}$ is a sum of elements in $\operatorname{sindec}\left(E_{8}\right)$ as $c_{1}-d_{8}$ is not. However, the usual subsum argument shows that $d_{2}=c_{2}-d_{8} \in$ $\operatorname{sindec}\left(E_{8}\right)$. As $c_{2,1}=1$ and $c_{2,1}^{\prime}=0$, so $d_{1}=c_{1}-d_{2}$.

Now

$$
\begin{aligned}
P_{3}:= & (300,30,-6,8,-1,-2,2,-1,0,3,0,2,30,3, \\
& 0,30,2,2,0,20,6,0,2)
\end{aligned}
$$


is in $\operatorname{indec}\left(E_{8}\right)$ whose values on 5-regular $(-1)$-regular classes are given. Clearly, $\phi\left(224_{s}\right):=224_{s}-\phi\left(16_{s}\right)$ is a modular spin character of degree 208 of $W\left(E_{8}\right)$, hence $\phi\left(224_{s}\right) \otimes P_{3}$, is a principal spin character of $W\left(E_{8}\right)$ and it contains

$$
c_{4}^{\prime}:=224_{s}+2592_{s}+9072_{s}+2.8192_{s}+2.2016_{s s}+896_{s}
$$

which is a sum of elements of $\operatorname{sindec}\left(E_{8}\right)$ in the principal block, as

$$
\begin{aligned}
\phi\left(244_{s} \otimes P_{3}\right)= & 224_{s}+2.8192_{s}+2.2016_{s s}+2.1120_{s}+11200_{s} \\
& +2800_{s}+2.6480_{s}+2592_{s}+9072_{s}+896_{s} .
\end{aligned}
$$

Thus at this stage, the matrix $R_{5}^{B_{2}}\left(E_{8}\right)$ is our next approximation for the decomposition matrix corresponding to the principal block. It contains all the simplified columns, $c_{4}$ and a new column $c_{4}^{\prime}$.

$$
R_{\overline{5}}^{B_{2}}\left(E_{8}\right)
$$

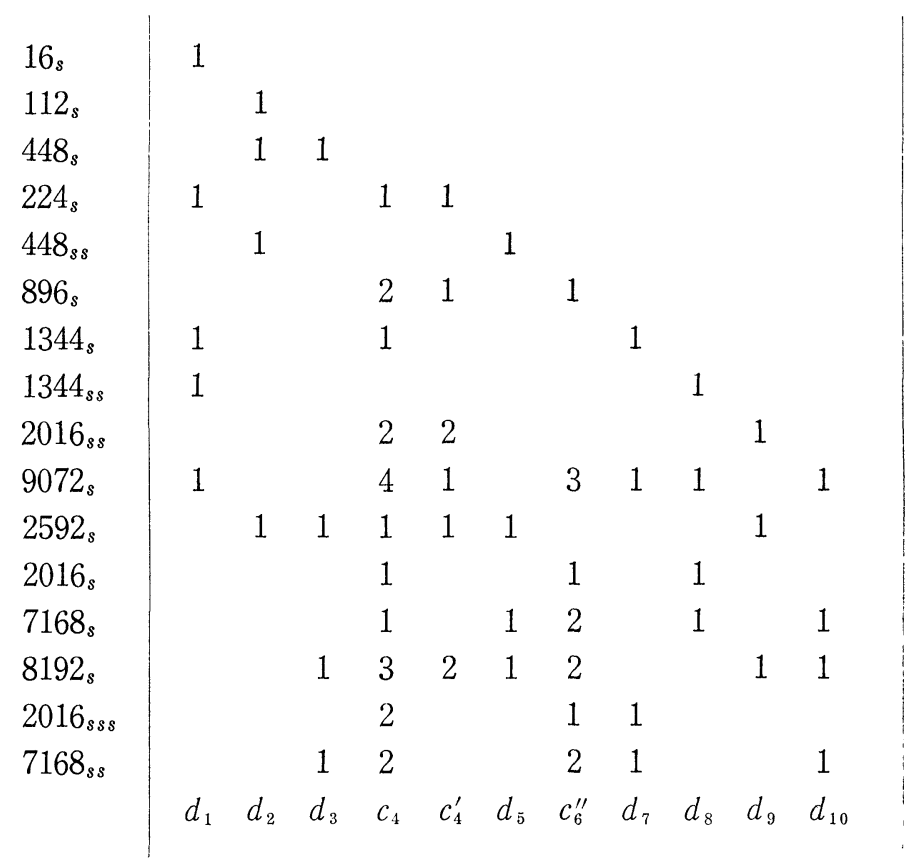

We must still resolve whether the following are possible:

$$
\begin{array}{ll}
\text { (i) } c_{4}^{\prime}-\alpha_{9} \cdot d_{9} & \text { where } \alpha_{9} \in[0,1] \\
\text { (ii) } c_{6}^{\prime \prime}-\alpha_{10} \cdot d_{10} & \text { where } \alpha_{10} \in[0,1,2] .
\end{array}
$$

We first show that $c_{4}^{\prime}-d_{9}$ is a principal spin character. This is equivalent 
to showing that there exist $\phi\left(2016_{s s}\right) \in \operatorname{sirm}\left(E_{8}\right)$, having degree 1808 corresponding to the 9 th column. We shall remove this ambiguity by showing that the character $\phi\left(2016_{s s}\right)$ with degree 1600 arising from the matrix $R_{5}^{B_{2}}\left(E_{8}\right)$ not a modular spin character. Now the product character $\phi\left(16_{s}\right) \otimes \phi\left(2016_{s s}\right)$ is a sum of elements in $\operatorname{irm}\left(E_{8}\right)$. However, these elements also occur without nonnegative coefficients, so it follows that $d_{4}=c_{4}^{\prime}-d_{9} \in \operatorname{sindec}\left(E_{8}\right)$.

We note that in addition to $d_{4}$, the column $c_{4}$ may contains $c_{6}^{\prime \prime}, d_{7}$ and $d_{9}$ once each. Comparing the coefficient of $9072_{s}$ in $c_{4}$, with the column $d_{4}, c_{6}^{\prime \prime}, d_{7}$ and $d_{9}$ implies that $d_{10}$ is a constituent of $c_{6}^{\prime \prime}$.

Hence

$$
d_{6}=c_{6}^{\prime \prime}-\alpha_{10} \cdot d_{10}, \quad \text { where } \alpha_{10} \in[1,2] .
$$

If $d_{6}=c_{6}^{\prime \prime}-d_{10}$, then there must exist $\phi\left(9072_{s}\right) \in \operatorname{sirm}\left(E_{8}\right)$, of degree 4816 which is associated with the last column. Now by computing the product $\phi\left(224_{s}\right) \otimes$ $\phi\left(9072_{s}\right)$ and then writing it in term of elements of $\operatorname{irm}\left(E_{8}\right)$, we see that $175_{P}$, $125_{n} \in \operatorname{irm}\left(E_{8}\right)$ having degrees divisible by $5^{2}$, occur with negative entries which is not true. Hence $c_{6}^{\prime \prime}-2 \cdot d_{10} \in \operatorname{sindec}\left(E_{8}\right)$ by the usual argument and we denote it by $d_{6}$. This completely determines the decomposition matrix $D_{5}\left(E_{8}\right)$ corresponding to the principal block and as given by:

$$
D_{5}\left(E_{8}\right)
$$

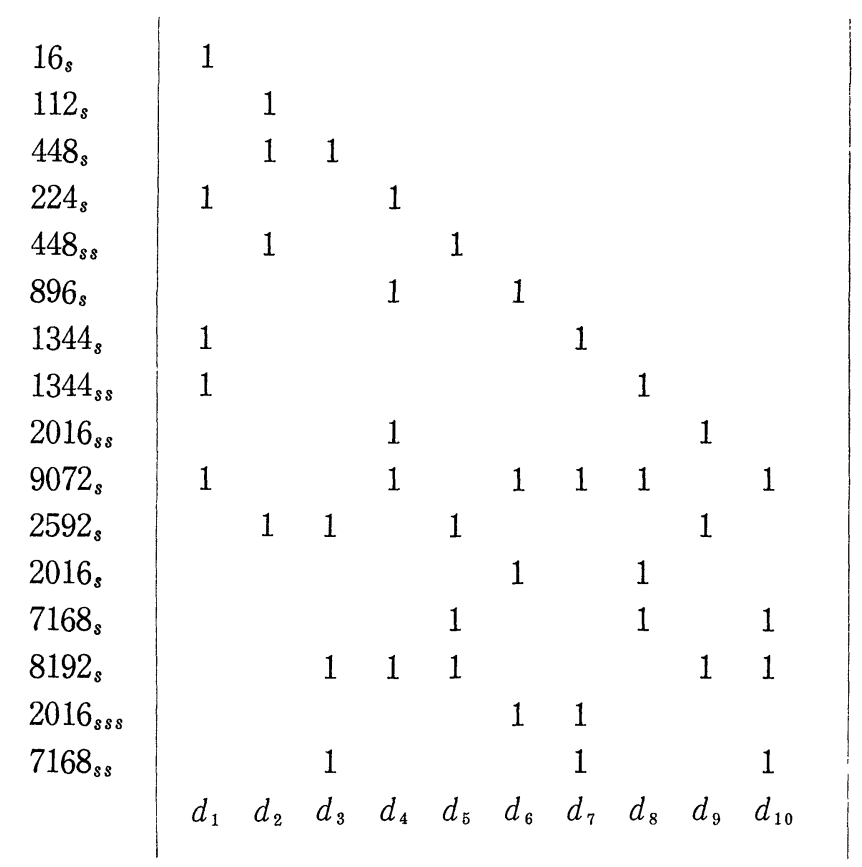




\section{4. $\boldsymbol{W}\left(\boldsymbol{E}_{7}\right), \boldsymbol{p}=7$}

Here $\left|\operatorname{sirm}\left(E_{7}\right)\right|=24$ and as the prime 7 divides the degrees of each of the following elements $168_{s}, 280_{s}, 112_{s}, 112_{s s}, 560_{s}, 448_{s}, 168_{r}, 280_{r}, 112_{r}, 112_{r r}, 560_{r}$ and $448_{r}$ of $\operatorname{sirr}\left(E_{7}\right)$, so each form its own block and therefore are in $\operatorname{sindec}\left(E_{7}\right)$.

There remains two other blocks (with full defect), each of which contains seven elements of $\operatorname{sirr}\left(E_{7}\right)$, namely:

$$
\begin{aligned}
& B_{1}: 8_{s}, 48_{s}, 512_{s}, 720_{s}, 120_{s}, 64_{s} \text { and } 64_{s s} . \\
& B_{2}: 8_{r}, 48_{r}, 512_{r}, 720_{r}, 120_{r}, 64_{r} \text { and } 64_{r r} .
\end{aligned}
$$

Since $8_{s}, 40_{s}, 72_{s}, 64_{s}, 64_{s s} \in \operatorname{indec}\left(E_{6}\right)$ and thus

$8_{s} \uparrow E_{7}=8_{s}+48_{s}+168_{s}+8_{r}+48_{r}+168_{r}$

$40_{s} \uparrow E_{7}=48_{s}+168_{s}+280_{s}+112_{s}+512_{s}+48_{r}+168_{r}+280_{r}+112_{r}+512_{r}$

$72_{s} \uparrow E_{7}=112_{s}+112_{s s}+512_{s}+720_{s}+560_{s}+112_{r}+112_{r r}+512_{r}+720_{r}+560_{r}$

$64_{s} \uparrow E_{7}=720_{s}+560_{s}+448_{s}+64_{s}+720_{r}+560_{r}+448_{r}+64_{r}$

$64_{s s} \uparrow E_{7}=720_{s}+560_{s}+448_{s}+64_{s s}+720_{r}+560_{r}+448_{r}+64_{r r}$.

Now blocks distribution of characters and characters degrees argument implies that the Brauer tree for $B_{1}$ is as follows:

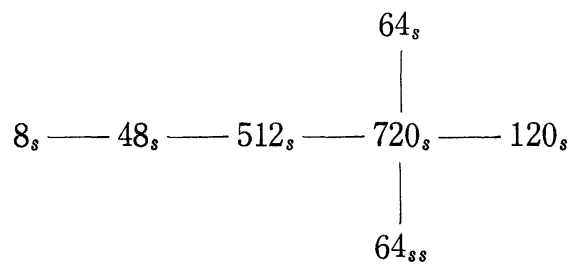

Similarly, the Brauer tree corresponding to the other block is obtained and is given below:

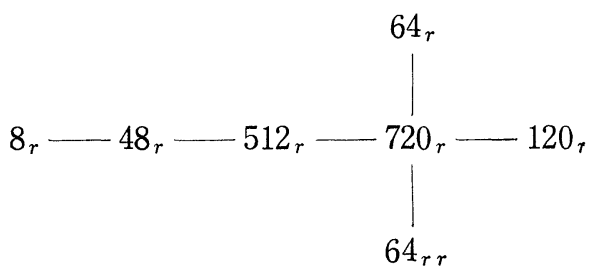

\section{5. $\boldsymbol{W}\left(\boldsymbol{E}_{8}\right), \boldsymbol{p}=7$}

There are twentynine 7-regular $\alpha$-regular classes in $W\left(E_{8}\right)$ so there are twentynine 7 -modular irreducible spin characters. 
Each of the irreducible spin character of $W\left(E_{8}\right)$, namely: $112_{s}, 448_{s}, 224_{s}$, $448_{s s}, 1680_{s}, 1344_{s}, 5600_{s}, 2016_{s}, 5600_{s s}, 9072_{s}, 2800_{s s}, 5600_{s s s}, 7168_{s}, 1120_{s}, 8400_{s}$, $11200_{s}, 6720_{s}, 2800_{s}, 1344_{s s}, 2016_{s s}, 2016_{s s s}, 7168_{s s}$ and $896_{s}$, whose degrees are divisible by 7 , from its own block.

All the remaining spin characters namely: $16_{s}, 320_{s}, 2592_{s}, 800_{s}, 4800_{s}, 6480_{s}$ and $8192_{s}$ of the group $W\left(E_{8}\right)$ belong to a single block of defect one.

From above section, we have that $\left(8_{s}+48_{s}\right), 112_{s}, 112_{s s}, 448_{s}$ and $\left(8_{s}+512_{s}\right)$ are in $\operatorname{sindec}\left(E_{7}\right)$. Then

$$
\begin{aligned}
& \left(8_{s}+48_{s}\right) \uparrow E_{8}=16_{s}+2.112_{s}+320_{s}+2.448_{s s}+1680_{s}+2016_{s}+5600_{s s}+2.1344_{s} . \\
& 112_{s} \uparrow E_{8}=320_{s}+448_{s}+1680_{s}+2592_{s}+5600_{s}+9072_{s}+7168_{s} . \\
& 112_{s s} \uparrow E_{8}=448_{s}+224_{s}+2592_{s}+1344_{s}+4800_{s}+8400_{s}+9072_{s} . \\
& 448_{s} \uparrow E_{8}=5600_{s}+4800_{s}+9072_{s}+898_{s}+2.8400_{s}+2.11200_{s}+6720_{s} \\
& \quad+6480_{s}+2.8192_{s}+2016_{s s}+2016_{s s s}+2.7168_{s s} . \\
& 280_{s} \uparrow E_{8}=2016_{s}+2.5600_{s s}+9072_{s}+800_{s}+2.5600_{s s s}+7168_{s} \\
& +11200_{s}+6720_{s}+1344_{s s}+6480_{s} .
\end{aligned}
$$

and

$$
\begin{aligned}
\left(48_{s}+512_{s}\right) \uparrow E_{8}=112_{s} & +320_{s}+448_{s}+2.1680_{s}+2592_{s}+5600_{s}+2.2016_{s} \\
+ & 3.5600_{s s}+2.9072_{s}+5600_{s s s}+2.7168_{s}+8400_{s} \\
+ & 2.11200_{s}+2.6720_{s}+2800_{s}+1344_{s s}+6480_{s}+8192_{s} .
\end{aligned}
$$

Now restricting these six decomposition to a block of nonzero defect of $W\left(E_{8}\right)$, we get the following matrix of principal characters:

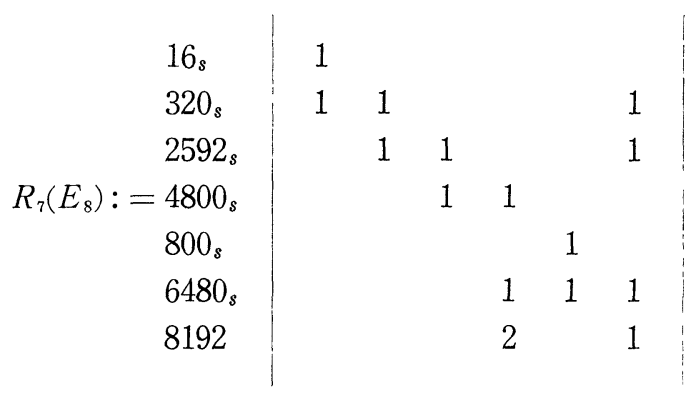

Since the characters 320 and 6480 are not 7 -conjugates and belong to the same block therefore they have no modular constituent in common. Hence we must subtract the second column from the last column. The same arguments implies that the last column must also be subtracted from the fourth column, since $4800_{s}$ and $6480_{s}$ are not 7 -conjugates. 
Hence the Brauer tree for this block is as follows:

$$
16_{s}-320_{s}-2592_{s}-4800_{s}-8192_{s}-6480_{s}-800_{s} \text {. }
$$

\section{Appendix}

$$
D_{5}\left(E_{7}\right)
$$

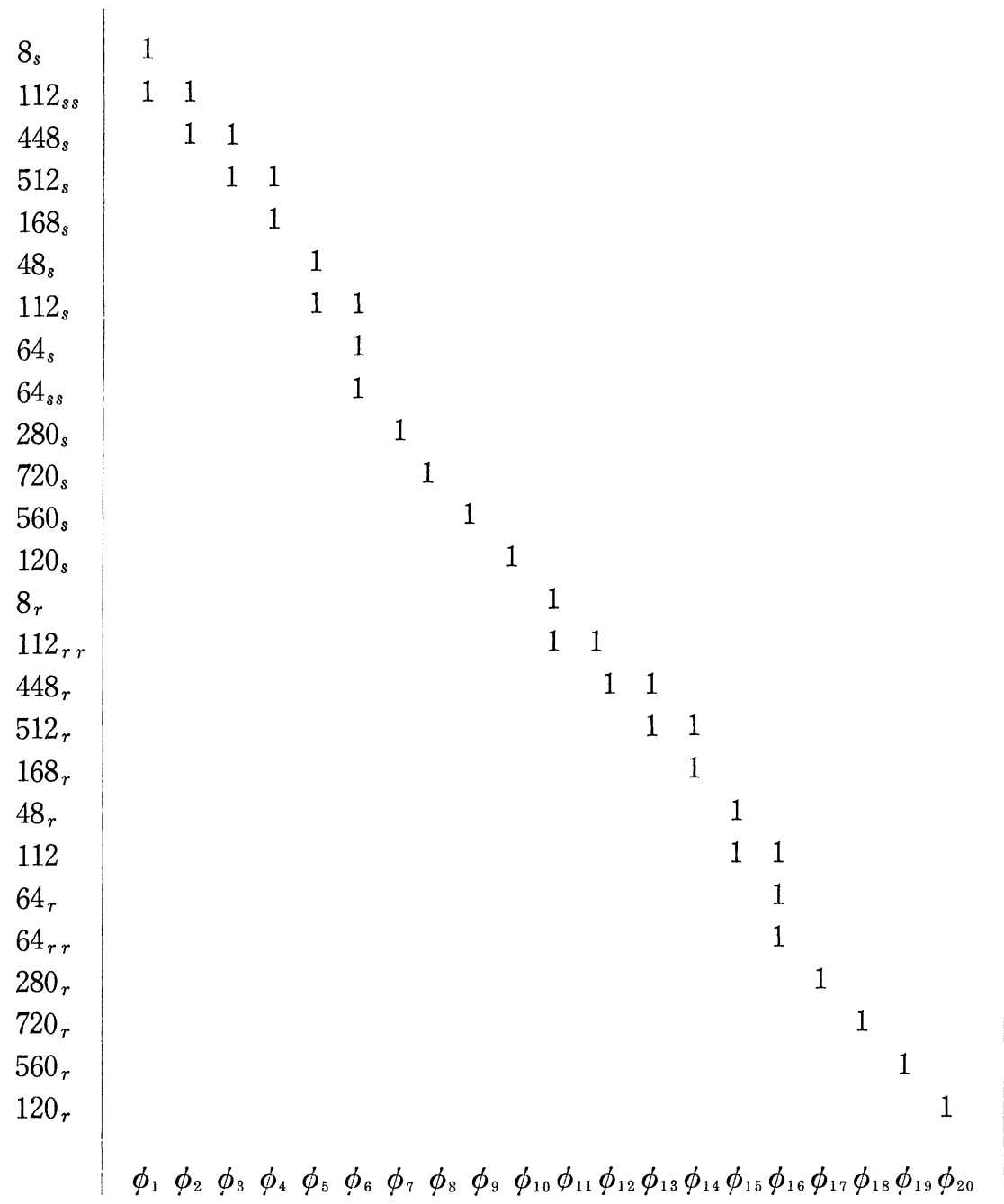




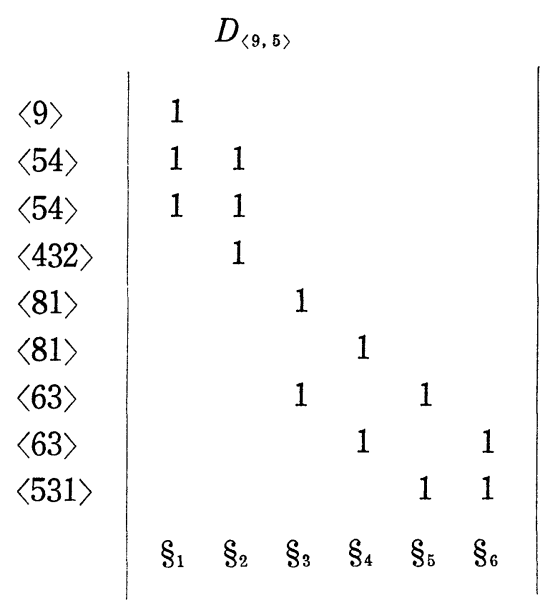

Acknowledgement. My thanks are due to Professor A. O. Morris for useful discussion during the preparation of this paper. Also I am grateful to the referee for his suggestions.

\section{REFERENCES}

[CR] C. W. Curtis And I. Reiner, Representation Theory of Finite Groups and Associate Algebra, Interscience pub. Co., New York, 1962.

[CCN] J.H. Conway, R. T. Curtis, S. P. Norton, R. A. Parker and R. A. Wilson, An Atlas of Finite Groups, Oxford Univ. Press, 1985.

[Fe] W. Feit, The Representation Theory of Finite Groups, North Holland, 1982.

[JK] G. D. JAmEs AND A. Kerber, The Representation Theory of the Symmetric Groups (Reading Massachussetts: Addison-Wesley, 1981).

[KM] F. KhosRaviYani ANd A. O. MorRis, Decomposition number of the exceptional Weyl groups II, J. Algebra, (2) 92 (1985), 525-531.

[Mo] A. O. MorRIs, Projective characters of exceptional Weyl groups, J. Algebra, 29 (1974), 567-586.

[Ya] A.K. YASEEN, Decomposition matrices for spin characters of symmetric groups II, to appear.

[Sa] M. SALEEM, The 3-modular spin characters of exceptional Weyl groups of type $E_{n}$, submitted.

Department of Mathematics

The University College of Wales

ABERYSTWYTH

DYFED SY23 3BZ

WALES

Present Address

Department of Mathematics

ISLAMIA UNIVERSITY

BAHAWALPUR

PAKISTAN 